\title{
On extendibility of additive code isometries
}

\author{
Serhii Dyshko * \\ Institut de mathématiques de Toulon, Université de Toulon, France
}

October 2, 2018

\begin{abstract}
For linear codes, the MacWilliams Extension Theorem states that each linear isometry of a linear code extends to a linear isometry of the whole space. But, in general, it is not the situation for nonlinear codes. In this paper it is proved, that if the length of an additive code is less than some threshold value, then an analogue of the MacWilliams Extension Theorem holds. One family of unextendible code isometries for the threshold value of code length is described.
\end{abstract}

\section{Introduction}

The main objective of the coding theory is to study the metric structure of a code. Therefore the classification of code isometries is vital for the completeness of the theory.

There is a full description of linear code isometries in a Hamming space. The famous MacWilliams Extension Theorem claims that each linear isometry of a linear code can be extended to a linear isometry of the full space.

The description of isometries in terms of extendibility is very convenient, because the classification of all isometries of the full space, both linear and nonlinear, have already been done, for example, in [1].

Unfortunately, in the case where the linearity of a code is not required, the situation is more complicated. There are nonlinear codes with isometries that do not extend to isometries of the whole space.

In general, it is a difficult task to describe codes, which have only extendible isometries. Nevertheless, considering some classes of codes, this problem can be solved in particular cases. For example, in [2], [3] and [4] authors described several families of nonlinear codes with all isometries extendible. There they also observed various classes of codes that have unextendible isometries. Among the studied families there are some subclasses of codes that achieve the Singleton bound (MDS codes, see [5, p. 20]), some subclasses of codes with equal distance between codewords (equidistant codes) and some perfect codes (see [5, Ch. §11]).

In this paper, we focus our attention on additive codes and the extendibility of additive code isometries. An additive code is a code that forms a group under addition. An additive isometry of an additive code is an isometry that is a group isomorphism. The importance of these codes is due to the fact that additive codes with additional requirement of a special kind of self-orthogonality naturally describe quantum stabilizer codes (see [6]).

The results presented in the paper are the following. Theorem 3 determines the threshold value of the code length for which an analogue of the MacWilliams Extension Theorem for additive codes holds. By providing Example 3, we proved that in general this result cannot be improved by increasing the bound on the code length.

\footnotetext{
*Electronic address: dyshko@univ-tln.fr
} 


\section{Additive codes and additive isometries}

Let $L^{m}$ be a Hamming space. There is a full description of linear isometries of linear codes in $L^{m}$. The map $f: L^{m} \rightarrow L^{m}$ is called monomial if it acts by permutation of coordinates and multiplications of coordinates by nonzero scalars.

Theorem 1 (MacWilliams Extension Theorem, see [7]). Let $C \subseteq L^{m}$ be a linear code. Each linear isometry of $C$ extends to a monomial map.

The MacWilliams Extension Theorem claims that any linear isometry of a linear code can be extended to a linear isometry of the whole space. A general analogue of the MacWilliams Extension Theorem does not exist for nonlinear codes. This means that there exists a nonlinear code and there exists an isometry of this code that does not extend to an isometry of the whole space. In [1] the author gives a full description of the isometries of the ambient space and in [2] there is given such an example of unextendible code isometry.

Theorem 2 (see [1]). Let $F$ be a finite set with at least two elements and let $m$ be a positive integer. A map $f: F^{m} \rightarrow F^{m}$ is an isometry if and only if there exist a permutation $\pi \in S_{m}$ and permutations $\sigma_{1}, \ldots, \sigma_{m} \in \operatorname{Sym}(F)$ such that for any $x=\left(x_{1}, \ldots, x_{m}\right) \in F^{m}$,

$$
f\left(\left(x_{1}, \ldots, x_{m}\right)\right)=\left(\sigma_{1}\left(x_{\pi(1)}\right), \ldots, \sigma_{m}\left(x_{\pi(m)}\right)\right) .
$$

Example 1 (see [2]). Suppose $F=\{0,1\}$. Two codes in $F^{4}$

$$
C=\{(0,0,0,0),(1,1,0,0),(1,0,1,0),(0,1,1,0)\}
$$

and

$$
D=\{(0,0,0,0),(1,1,0,0),(1,0,1,0),(1,0,0,1)\}
$$

are isometric, i.e. there exists an isometry $f: C \rightarrow D$. Indeed, in both codes the distance between two different codewords is 2, thus any bijection $f: C \rightarrow D$ is an isometry. For any position, there exists two different codewords in $D$ that have different values in this position. But all the codewords in $C$ have equal values on the fourth position. According to Theorem 2, any isometry between these two codes cannot be extended to an isometry of the space $F^{4}$.

As we have already noted in the introduction, the studying of the extendibility property for code isometries in general is difficult and only a few families of codes and their isometries have been properly described. In this paper we focus our attention on the extendibility of additive code isometries.

A code in $L^{m}$ is called additive if it is an additive subgroup of $L^{m}$. An additive isometry of an additive code $C$ is an isometry that is a group homomorphism. Evidently, a map $f$ is an additive isometry if and only if $f$ preserves the Hamming weight.

Let $K$ be a subfield of $L$. Along with additive codes we will speak about $K$-linear codes, i.e. codes that are $K$-linear subspaces of $L^{m}$. The notions of additive and $K$-linear codes in $L^{m}$ are in some sense equivalent. Any $K$-linear code is additive and, in the other way, any additive code is $\mathbb{F}_{p}$-linear, where $p$ is the characteristic of $L$. If $K=L$, a $K$-linear code is linear. Obviously, any $K$-linear isometry is additive and any additive isometry is $\mathbb{F}_{p}$-linear. 
Example 2. Consider two codes $C_{1}=\left\{(0,0,0),(1,1,0),(\omega, 0,1),\left(\omega^{2}, 1,1\right)\right\}$ and $C_{2}=\left\{(0,0,0),\left(0, \omega^{2}, \omega\right),(1,0,1),\left(1, \omega^{2}, \omega^{2}\right)\right\}$ in $\mathbb{F}_{4}^{3}$, where $\mathbb{F}_{4}=\left\{0,1, \omega, \omega^{2}\right\}$ and $\omega+1=\omega^{2}$. All the codes are $\mathbb{F}_{2}$-linear. Define a map $f: C_{1} \rightarrow C_{2}$ in the following way: $f((0,0,0))=(0,0,0), f((1,1,0))=\left(0, \omega^{2}, \omega\right), f((\omega, 0,1))=$ $(1,0,1)$ and $f\left(\left(\omega^{2}, 1,1\right)\right)=\left(1, \omega^{2}, \omega^{2}\right)$. Evidently, the map $f$ is $\mathbb{F}_{2}$-linear and it preserves the Hamming weight. Therefore $f$ is an $\mathbb{F}_{2}$-linear isometry of the $\mathbb{F}_{2}$-linear code $C_{1}$ in $\mathbb{F}_{4}^{3}$. Both codes $C_{1}$ and $C_{2}$ are not $\mathbb{F}_{4}$-linear.

Our main objects of study are the $K$-linear isometries of $K$-linear codes. We begin with the description of all $K$-linear isometries of $L^{m}$. The field $L$ can be observed as a finite-dimensional vector space over $K$. By $\operatorname{Aut}_{K}(L)$ we denote the group of all $K$-linear invertible maps from $L$ to itself.

Definition 1. A map $f: L^{m} \rightarrow L^{m}$ is called $K$-monomial if there exist a permutation $\pi \in S_{m}$ and automorphisms $g_{1}, \ldots, g_{m} \in \operatorname{Aut}_{K}(L)$ such that for all $u \in L^{m}$,

$$
f(u)=f\left(\left(u_{1}, u_{2}, \ldots, u_{m}\right)\right)=\left(g_{1}\left(u_{\pi(1)}\right), g_{2}\left(u_{\pi(2)}\right), \ldots, g_{m}\left(u_{\pi(m)}\right)\right) .
$$

Proposition 1. A map $f: L^{m} \rightarrow L^{m}$ is K-monomial if and only if it is a $K$-linear isometry.

Proof. The only if part is obvious. In the other direction, use Theorem 2. Since $K$-linear permutations of $L$ are exactly elements of $\operatorname{Aut}_{K}(L)$, any $K$ linear isometry is a $K$-monomial map.

We call a $K$-linear code isometry extendible if it is a restriction of a $K$ monomial map on the code. Otherwise, we call it unextendible. The following example shows an unextendible additive code isometry.

Example 3. Let $K \subset L$ be a pair of finite fields, $m=|K|+1$ and $\omega \in L \backslash K$. Consider two $K$-linear codes $C_{1}=\left\langle v_{1}, v_{2}\right\rangle_{K}$ and $C_{2}=\left\langle u_{1}, u_{2}\right\rangle_{K}$ in $L^{m}$ with

$$
\left(\begin{array}{l}
v_{1} \\
v_{2}
\end{array}\right)=\left(\begin{array}{ccccc}
1 & 1 & \ldots & 1 & 0 \\
x_{1} & x_{2} & \ldots & x_{|K|} & 1
\end{array}\right) \stackrel{f}{\rightarrow}\left(\begin{array}{ccccc}
1 & 1 & \ldots & 1 & 0 \\
\omega & \omega & \ldots & \omega & 0
\end{array}\right)=\left(\begin{array}{l}
u_{1} \\
u_{2}
\end{array}\right),
$$

where $x_{i} \in K$ are all different. The $K$-linear map $f: C_{1} \rightarrow C_{2}$, defined by $f\left(v_{1}\right)=u_{1}$ and $f\left(v_{2}\right)=u_{2}$, is an isometry. Indeed, let $\alpha v_{1}+\beta v_{2}$ be an arbitrary element in $C_{1} \backslash\{0\}$, where $\alpha, \beta \in K$. If $\beta=0$, then $\operatorname{wt}\left(\alpha v_{1}+\beta v_{2}\right)=m-1$. If $\beta \neq 0$ then the equation $\alpha+\beta x_{i}=0$, where $i \in\{1, \ldots,|K|\}$, has exactly one solution $x_{i}=-\alpha \beta^{-1} \in K$ and thus $\operatorname{wt}\left(\alpha v_{1}+\beta v_{2}\right)=m-1$. Therefore, all nonzero elements in $C_{1}$ have the weight equal to $m-1$. It is easy to see that all nonzero codewords in $C_{2}$ also have the weight $m-1$. The map $f$ maps nonzero elements of $C_{1}$ to nonzero elements of $C_{2}$ and hence is an isometry. At the same time, there is no $K$-monomial map that acts on $C_{1}$ in the same way as $f$. The last coordinates of all vectors in $C_{2}$ are always zero, but there is no such all-zero coordinate in $C_{1}$.

\section{Column spaces}

Let $K$ be a finite field and let $U$ and $L$ be $K$-linear vector spaces over $K$ of dimensions $k$ and $n$ respectively. Fix bases in $U$ and $L$ and let $b_{1}, \ldots, b_{n}$ be 
a basis of $L$ over $K$. For simplicity assume that $L$ is a finite field and $K$ is a subfield of $L$.

Denote by $\mathrm{M}_{a \times b}(F)$ the set of all $a \times b$ matrices with the entries from a field $F$. Let $A \in \mathrm{M}_{k \times n}(L)$ be a matrix and let $v \in L^{k}$ be a column of $A$. Suppose $v_{1}, \ldots, v_{n} \in U$ is the expansion of $v$ in the basis $b_{1}, \ldots, b_{n}$. This means that $v=\sum_{i=1}^{n} b_{i} v_{i}$, where the multiplication is component-wise. Define a column space $V \subseteq U$ of the vector $v$ as the $K$-linear span $V=\left\langle v_{1}, \ldots, v_{n}\right\rangle_{K}$. The definition of a column space does not depend on the choice of a basis of $L$ over $K$. Call $\mathcal{V}=\left(V_{1}, \ldots, V_{m}\right)$ the tuple of spaces of $A$, where $V_{i}$ denotes the column space of $i$ th column of $A$, for $i \in\{1, \ldots, m\}$.

Example 4. Consider the finite field $\mathbb{F}_{4}=\left\{0,1, \omega, \omega^{2}\right\}$, where $\omega+1=\omega^{2}$. The matrix $A \in \mathrm{M}_{3 \times 3}\left(\mathbb{F}_{4}\right)$,

$$
A=\left(\begin{array}{lll}
1 & 1 & 0 \\
\omega & \omega & 0 \\
1 & 0 & 1
\end{array}\right)
$$

has the following expansion of columns in the $\mathbb{F}_{2}$-linear basis $1, \omega$ of $\mathbb{F}_{4}$,

$$
\left(\begin{array}{l}
1 \\
\omega \\
1
\end{array}\right)=\left(\begin{array}{l}
1 \\
0 \\
1
\end{array}\right)+\omega\left(\begin{array}{l}
0 \\
1 \\
0
\end{array}\right) ;\left(\begin{array}{l}
1 \\
\omega \\
0
\end{array}\right)=\left(\begin{array}{l}
1 \\
0 \\
0
\end{array}\right)+\omega\left(\begin{array}{l}
0 \\
1 \\
0
\end{array}\right) ;\left(\begin{array}{l}
0 \\
0 \\
1
\end{array}\right)=\left(\begin{array}{l}
0 \\
0 \\
1
\end{array}\right)+\omega\left(\begin{array}{l}
0 \\
0 \\
0
\end{array}\right) \text {. }
$$

The column spaces $V_{1}, V_{2}, V_{3} \subseteq \mathbb{F}_{2}^{3}$ are: $V_{1}=\langle(1,0,1),(0,1,0)\rangle_{\mathbb{F}_{2}}, V_{2}=\langle(1,0,0),(0,1,0)\rangle_{\mathbb{F}_{2}}$ and $V_{3}=\langle(0,0,1)\rangle_{\mathbb{F}_{2}}$.

For two vector spaces $U, L$ over a field $K$ we denote by $\operatorname{Hom}_{K}(U, L)$ the set of all $K$-linear maps from $U$ to $L$.

Suppose $\sigma \in \operatorname{Hom}_{K}(U, L)$. There exists a unique matrix $M \in \mathrm{M}_{k \times n}(K)$ such that for all $a \in U, \sigma(u)=M^{T} u$. Define the dual map $\sigma^{*} \in \operatorname{Hom}_{K}(L, U)$ as $\sigma^{*}(b)=M b$ for all $b \in L$. Evidently, $\sigma^{* *}=\sigma$. Let $X$ be another vector space over $K$. Suppose $\sigma_{1} \in \operatorname{Hom}_{K}(U, L)$ and $\sigma_{2} \in \operatorname{Hom}_{K}(L, X)$. Then $\left(\sigma_{2} \sigma_{1}\right)^{*}=$ $\sigma_{1}^{*} \sigma_{2}^{*}$. Note that if $g \in \operatorname{Aut}_{K}(L)$, then also $g^{*} \in \operatorname{Aut}_{K}(L)$.

The matrix $A \in \mathrm{M}_{k \times m}(L)$ naturally defines a map $\lambda \in \operatorname{Hom}_{K}\left(U, L^{m}\right)$, $\lambda(u)=A^{T} u$, where $u \in U$. We present $\lambda$ in the form $\lambda=\left(\lambda_{1}, \ldots, \lambda_{m}\right)$, where $\lambda_{i}(u)$ is the projection of $\lambda(u)$ on $i$ th coordinate, $i \in\{1, \ldots, m\}, u \in U$. Obviously, $\lambda_{i} \in \operatorname{Hom}_{K}(U, L)$, for $i \in\{1, \ldots, m\}$, and it corresponds to the $i$ th column of $A$. One can see that for all $i \in\{1, \ldots, m\}, \lambda_{i}^{*}(L)=V_{i}$, where $\mathcal{V}=\left(V_{1}, \ldots, V_{m}\right)$ is the tuple of spaces of $A$.

Let $f: \lambda(U) \rightarrow L^{m}$ be a $K$-linear map. Define a map $\mu=f \lambda \in \operatorname{Hom}_{K}\left(U, L^{m}\right)$. The following diagram is commutative,

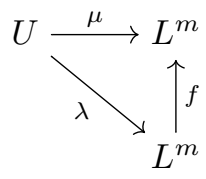

Suppose $A^{\prime} \in \mathrm{M}_{k \times m}(L)$ is such that $\mu(u)=A^{\prime T} u$ for all $u \in U$. Let $\mathcal{U}=$ $\left(U_{1}, \ldots, U_{m}\right)$ be the tuple of spaces of $A^{\prime}$. Note that for any $i \in\{1, \ldots, m\}$, $U_{i}=\mu_{i}^{*}(L)$.

Call two tuples of spaces $\mathcal{U}=\left(U_{1}, \ldots, U_{m}\right)$ and $\mathcal{V}=\left(V_{1}, \ldots, V_{m}\right)$ equivalent and denote $\mathcal{U} \sim \mathcal{V}$, if there exists a permutation $\pi \in S_{m}$ such that $V_{i}=U_{\pi(i)}$ for all $i \in\{1, \ldots, m\}$. 
Lemma 1. Let $\sigma, \tau \in \operatorname{Hom}_{K}(U, L)$. There exists $g \in \operatorname{Aut}_{K}(L)$ such that $\sigma=g \tau$ if and only if $\sigma^{*}(L)=\tau^{*}(L)$.

Proof. The $K$-linear spaces $\sigma^{*}(L)$ and $\tau^{*}(L)$ are equal if and only if there exists a map $h \in \operatorname{Aut}_{K}(L)$ such that $\tau^{*} h=\sigma^{*}$, or the same, calculating the dual of both maps, there exists a map $g=h^{*} \in \operatorname{Aut}_{K}(L)$ such that $g \tau=\sigma$.

Proposition 2. The K-linear map $f$ is extendible if and only if the tuples of spaces $\mathcal{V}$ and $\mathcal{U}$ are equivalent.

Proof. The map $f$ is extendible if and only if there exist a permutation $\pi \in S_{m}$ and maps $g_{1}, \ldots, g_{m} \in \operatorname{Aut}_{K}(L)$ such that $\mu_{i}=g_{i} \lambda_{\pi(i)}$, for all $i \in\{1, \ldots, m\}$. From Lemma 1, the last statement is equivalent to the existence of a permutation $\pi \in S_{m}$ such that $U_{i}=V_{\pi(i)}$.

\section{Characters and their applications}

The proof of the MacWilliams Extension Theorem firstly appeared in the works of MacWilliams and it was later refined by several authors. Namely, in [7], Ward and Wood greatly simplified it, using a character theory approach. Generalized analogues of the MacWilliams Extension Theorem for the codes linear over rings and the related properties were discussed in [8], [9] and [10] where the authors also used the techniques of the character theory.

Recall the notation and basic properties of characters (for more details see [11, Ch. $18 \S 2]$, [5, Ch. $5 \S 4]$ and [7]). For a finite abelian group $G$ let $\hat{G}$ be the set of all homomorphisms from $(G,+)$ to $\left(\mathbb{C}^{*}, \times\right)$, where $\mathbb{C}^{*}$ is the multiplicative group of complex numbers. With the defined sum of homomorphisms: for $g, h \in \hat{G}, x \in G,(g+h)(x)=g(x) h(x)$, the set $\hat{G}$ form an abelian group and is called a group of characters. It is proved, that the groups $(G,+)$ and $(\hat{G},+)$ are isomorphic (see [7]).

Let $G$ be a $K$-linear space of dimension $k$. Fix a $K$-linear basis in $G$ and consider the bilinear form $(-,-)_{G}: G \times G \rightarrow K$, for any $x, y \in G,(x, y)_{G}=$ $\sum_{i=1}^{k} x_{i} y_{i}$. Let $\pi$ be a nontrivial character in $K$. Define a map $\psi_{G}: G \rightarrow \hat{G}$ as $\psi_{G}(x)(y)=\chi_{x}(y)=\pi\left((x, y)_{G}\right)$, where, $x, y \in G$. Define in $\hat{G}$ a multiplication by scalar $(\lambda g)(x)=g(\lambda x)$, where $x \in G, g \in \hat{G}, \lambda \in K$. It is easy to see that $\hat{G}$ is a vector space over $K$ and the map $\psi_{G}$ is an isomorphism of $K$-linear spaces.

The important property of characters is their linear independence as complex functions. If $\chi_{1}, \ldots, \chi_{k} \in \hat{G}$ are different characters and $a_{1}, \ldots, a_{k} \in \mathbb{C}$, then the equality, for all $x \in G, \sum_{i=1}^{k} a_{i} \chi_{i}(x)=0$, implies that all $a_{i}=0$ (see [11, p. 283]).

Also, it is a well known fact that the weight function can be rewritten as a sum of characters (see [5, p. 143]). For the weight function wt : $G \rightarrow\{0,1\}$, that maps 0 to 0 and other elements to 1 , the following holds, for all $a \in G$,

$$
\frac{1}{|G|} \sum_{\chi \in \hat{G}} \chi(a)=\frac{1}{|G|} \sum_{b \in G} \chi_{b}(a)=1-\operatorname{wt}(a) .
$$

Recall that $U$ and $L$ are vector spaces over $K$. Let $\sigma$ be an element in $\operatorname{Hom}_{K}(U, L)$. Define a map $\hat{\sigma}: \hat{L} \rightarrow \hat{U}$ as $\hat{\sigma}(\chi)=\chi \sigma$, for all $\chi \in \hat{L}$. The map $\hat{\sigma}$ is a $K$-linear homomorphism. Indeed, for any $\chi_{1}, \chi_{2} \in \hat{L}, u \in U$, 
$\left(\hat{\sigma}\left(\chi_{1}+\chi_{2}\right)\right)(u)=\chi_{1}(\sigma(u)) \chi_{2}(\sigma(u))=\left(\hat{\sigma}\left(\chi_{1}\right)+\hat{\sigma}\left(\chi_{2}\right)\right)(u)$ and for any $\chi \in \hat{L}$, $\lambda \in K, u \in U, \hat{\sigma}(\lambda \chi)(u)=\chi(\lambda \sigma(u))=\chi(\sigma(\lambda u))=(\lambda \hat{\sigma}(\chi))(u)$.

Lemma 2. For each $\sigma \in \operatorname{Hom}_{K}(U, L)$ the following diagram is commutative,

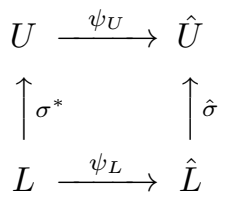

Proof. For $b \in L$ calculate $\hat{\sigma}\left(\psi_{L}(b)\right)=\chi_{b} \sigma$ and $\psi_{U}\left(\sigma^{*}(b)\right)=\chi_{\sigma^{*}(b)}$. Let matrix $M \in \mathrm{M}_{k \times n}(K)$ be such that $\sigma(u)=M^{T} u$ and $\sigma^{*}(b)=M b$ for $u \in U, b \in$ $L$. For all $u \in U, \chi_{b}(\sigma(u))=\chi_{b}\left(M^{T} u\right)=\pi\left(\left(b, M^{T} u\right)_{L}\right)=\pi\left(b^{T} M^{T} u\right)=$ $\pi\left((M b, u)_{U}\right)=\chi_{\sigma^{*}(b)}(u)$.

Let $X$ be a set and let $Y$ be a subset of $X$. An indicator function is a map $\mathbb{1}_{Y}: X \rightarrow\{0,1\}$, such that $\mathbb{1}_{Y}(x)=1$ if $x \in Y$ and $\mathbb{1}_{Y}(x)=0$ otherwise. Recall that for a map $\lambda \in \operatorname{Hom}_{K}\left(U, L^{m}\right)$ by $\lambda_{i}$ we denote the projection of $\lambda$ on the $i$ th coordinate and $V_{i}=\lambda_{i}^{*}(L)$, where $i \in\{1, \ldots, m\}$.

Proposition 3. Let $\lambda \in \operatorname{Hom}_{K}\left(U, L^{m}\right)$. For any $u \in U$ the following equality holds,

$$
\operatorname{wt}(\lambda(u))=m-\sum_{v \in U}\left(\sum_{i=1}^{m} \frac{1}{\left|V_{i}\right|} \mathbb{1}_{V_{i}}(v)\right) \chi_{v}(u) .
$$

Proof. For any $u \in U$,

$$
\begin{array}{r}
m-\operatorname{wt}(\lambda(u))=\sum_{i=1}^{m}\left(1-\operatorname{wt}\left(\lambda_{i}(u)\right)\right)=\sum_{i=1}^{m} \frac{1}{|L|} \sum_{\chi \in \hat{L}} \chi\left(\lambda_{i}(u)\right) \\
=\frac{1}{|L|} \sum_{i=1}^{m} \sum_{\chi \in \hat{L}} \hat{\lambda_{i}}(\chi)(u)=\frac{1}{|L|} \sum_{i=1}^{m} \sum_{\pi \in \hat{U}}\left(\left|\operatorname{Ker} \hat{\lambda}_{i}\right| \mathbb{1}_{\hat{\lambda}_{i}(\hat{L})}(\pi)\right) \pi(u) \\
=\sum_{\pi \in \hat{U}}\left(\sum_{i=1}^{m} \frac{1}{\mid \hat{\lambda_{i}(\hat{L}) \mid}} \mathbb{1}_{\hat{\lambda_{i}(\hat{L})}}(\pi)\right) \pi(u) .
\end{array}
$$

Substitute $\pi \in \hat{U}$ by $\psi_{U}(v)$, for $v \in U$. Consider the fact that $\psi_{U}(v)=\chi_{v} \in \hat{U}$. Lemma 2 implies $\psi_{U}^{-1} \hat{\lambda_{i}}(\hat{L})=\lambda_{i}^{*} \psi_{L}^{-1}(\hat{L})=\lambda_{i}^{*}(L)=V_{i}$, hence $\mathbb{1}_{\hat{\lambda_{i}}(\hat{L})} \psi_{U}=$ $\mathbb{1}_{\psi_{U}^{-1} \hat{\lambda_{i}(\hat{L})}}=\mathbb{1}_{V_{i}}$ and $\left|\hat{\lambda_{i}}(\hat{L})\right|=\left|V_{i}\right|$, for $i \in\{1, \ldots, m\}$.

\section{The main theorem}

Let $K \subseteq L$ be a pair of finite fields. We use the representation of the weight function presented in Proposition 3 to get a description of $K$-linear isometries of $K$-linear codes in $L^{m}$.

Let $C$ be a $K$-linear code in $L^{m}$ with some fixed $K$-linear basis. The matrix $A \in \mathrm{M}_{k \times m}(L)$, with the rows equal to the basis vectors of $C$, is called a generator matrix of $C$. Let $\mathcal{V}=\left(V_{1}, \ldots, V_{m}\right)$ the tuple of spaces of $A$. Call $\mathcal{V}$ a tuple of spaces of $C$. Since a generator matrix of a code is not unique, a tuple of spaces of a code is also not unique. 
Proposition 4. Let $C$ be a $K$-linear code and $\left(V_{1}, \ldots, V_{m}\right)$ be a tuple of spaces of $C$. The equality $\operatorname{dim}_{K} C=\operatorname{dim}_{K}\left(\sum_{i=1}^{m} V_{i}\right)$ holds.

Proof. Let $A \in \mathrm{M}_{k \times m}(L)$ be a matrix that correspond to the tuple of spaces $\left(V_{1}, \ldots, V_{m}\right)$, i.e. $V_{i}$ is a column space of the $i$ th column $v_{i} \in L^{k}$ of $A$, for all $i \in\{1, \ldots, m\}$. Fix a $K$-linear basis $b_{1}, \ldots, b_{n}$ of $L$ over $K$ and denote by $v_{i j} \in U$ the $j$ th term of the expansion of $v_{i}$ in the basis, for all $i \in\{1, \ldots, m\}, j \in$ $\{1, \ldots, n\}$. Denote $B \in \mathrm{M}_{k \times n m}(K)$ the matrix formed by $n m$ columns $v_{i j}$, $i \in\{1, \ldots, m\}, j \in\{1, \ldots, n\}$. The row rank of $B$ equals to the row rank of $A$ and is equal to $\operatorname{dim}_{K} C$. From the other side, the column rank of $B$ equals to the dimension of the column space of matrix $B$ and is equal to $\operatorname{dim}_{K} \sum_{i=1}^{m} V_{i}$.

Let $f: C \rightarrow L^{m}$ be a $K$-linear map. Let $U$ be a vector space over $K$ with the dimension equal to $\operatorname{dim}_{K} C$. Denote by $\lambda$ the map in $\operatorname{Hom}_{K}\left(U, L^{m}\right)$ defined as $\lambda(u)=A^{T} u$, for $u \in U$. Since $\lambda(U)=C$, we can define a map $\mu=f \lambda \in \operatorname{Hom}_{K}\left(U, L^{m}\right)$. Let $A^{\prime} \in \mathrm{M}_{k \times m}(L)$ be such that $\mu(u)=A^{\prime T} u$, for all $u \in U$. Denote by $\mathcal{U}=\left(U_{1}, \ldots, U_{m}\right)$ and $\mathcal{V}=\left(V_{1}, \ldots, V_{m}\right)$ tuples of spaces of matrices $A^{\prime}$ and $A$ correspondingly.

Proposition 5. Let $C$ be a $K$-linear code in $L^{m}$ and $f: C \rightarrow L^{m}$ be a $K$-linear map. The map $f$ is an isometry if and only if

$$
\sum_{i=1}^{m} \frac{1}{\left|V_{i}\right|} \mathbb{1}_{V_{i}}=\sum_{i=1}^{m} \frac{1}{\left|U_{i}\right|} \mathbb{1}_{U_{i}} .
$$

Proof. By definition, a map $f$ is an isometry if for all $x \in C$, $\operatorname{wt}(x)=\operatorname{wt}(f(x))$, or the same for a $K$-linear map $f$, for all $u \in U$, wt $(\lambda(u))=\operatorname{wt}(\mu(u))$. Consequently, using Proposition 3, $f$ is an isometry if and only if the following equality of functions holds,

$$
\sum_{v \in U}\left(\sum_{i=1}^{m} \frac{1}{\left|V_{i}\right|} \mathbb{1}_{V_{i}}(v)\right) \chi_{v}=\sum_{v \in U}\left(\sum_{i=1}^{m} \frac{1}{\left|U_{i}\right|} \mathbb{1}_{U_{i}}(v)\right) \chi_{v} .
$$

Since different characters in $\hat{U}$ are linearly independent, the coefficients in the equation are equal for each $v \in U$.

Proposition 5 shows that the task of description of $K$-linear isometries can be reformulated in terms of solutions of eq. (1), where $U_{1}, \ldots, U_{m}, V_{1}, \ldots, V_{m}$ are spaces in $U$, and dimensions of all spaces are bounded by $n$. We call the couple of tuples of spaces $(\mathcal{U}, \mathcal{V})$ the solution, if $\mathcal{U}$ and $\mathcal{V}$ satisfy eq. (1).

Evidently, if $\mathcal{U} \sim \mathcal{V}$, then $(\mathcal{U}, \mathcal{V})$ is a solution. Call a solution $(\mathcal{U}, \mathcal{V})$ trivial if $\mathcal{U} \sim \mathcal{V}$ and nontrivial otherwise. To illustrate Proposition 2, Proposition 5 and give an example of a nontrivial solution, we consider the following example observed in [12].

Example 5. Let the field $\mathbb{F}_{4}=\left\{0,1, \omega, \omega^{2}\right\}$ be generated by $\omega^{2}=\omega+1$. Define an $\mathbb{F}_{2}$-linear map $f: C \rightarrow \mathbb{F}_{4}^{3}$ on the generators in the following way: $f((1,1,0))=(1,1,0), f((\omega, \omega, 0))=(1,0,1)$ and $f((1,0,1))=(\omega, \omega, 0)$. Consider the following generator matrix $A$ of $C$ and the corresponding generator matrix $A^{\prime}$ of $f(C)$,

$$
A=\left(\begin{array}{lll}
1 & 1 & 0 \\
\omega & \omega & 0 \\
1 & 0 & 1
\end{array}\right) \stackrel{f}{\rightarrow}\left(\begin{array}{lll}
1 & 1 & 0 \\
1 & 0 & 1 \\
\omega & \omega & 0
\end{array}\right)=A^{\prime}
$$


Calculate the tuples of spaces $V_{1}, V_{2}, V_{3} \subseteq \mathbb{F}_{2}^{3}$ and $U_{1}, U_{2}, U_{3} \subseteq \mathbb{F}_{2}^{3}$. The spaces are: $V_{1}=\langle(1,0,1),(0,1,0)\rangle_{\mathbb{F}_{2}}, V_{2}=\langle(1,0,0),(0,1,0)\rangle_{\mathbb{F}_{2}}$ and $V_{3}=\langle(0,0,1)\rangle_{\mathbb{F}_{2}}$. In the same way, $U_{1}=\langle(1,1,0),(0,0,1)\rangle_{\mathbb{F}_{2}}, U_{2}=\langle(1,0,0),(0,0,1)\rangle_{\mathbb{F}_{2}}$ and $U_{3}=$ $\langle(0,1,0)\rangle_{\mathbb{F}_{2}}$. The defined spaces $V_{1}, V_{2}, V_{3}$ and $U_{1}, U_{2}, U_{3}$ satisfy the equation,

$$
\mathbb{1}_{V_{1}}+\mathbb{1}_{V_{2}}+2 \mathbb{1}_{V_{3}}=\mathbb{1}_{U_{1}}+\mathbb{1}_{U_{2}}+2 \mathbb{1}_{U_{3}},
$$

and therefore satisfy eq. (1). By Proposition 5, the map $f: C \rightarrow \mathbb{F}_{4}^{3}$ is an $\mathbb{F}_{2}$-linear isometry. Moreover, by Proposition 2 , since the tuples $\left(V_{1}, V_{2}, V_{3}\right)$ and $\left(U_{1}, U_{2}, U_{3}\right)$ are not equivalent, the isometry $f$ is unextendible.

Combining Proposition 2 and Proposition 5, we claim that a $K$-linear isometry is extendible if and only if the corresponding solution of eq. (1) is trivial. Nontrivial solutions of the equation must satisfy specific requirements on the subspace coverings. Such coverings and related questions are discussed in [13] and are partially connected with our results.

Lemma 3. Let $V$ be a nonzero vector space over $K$ and let $U_{i} \subset V$ be proper subspaces, for $i \in\{1, \ldots, m\}$. If $V=\bigcup_{i=1}^{m} U_{i}$, then $m$ is greater than the cardinality of $K$.

Proof. For any $i \in\{1, \ldots, m\}, \operatorname{dim}_{K} U_{i} \leq \operatorname{dim}_{K} V-1$ and hence $\left|U_{i}\right| \leq \frac{|V|}{|K|}$. Thus we have

$$
|V|<\sum_{i=1}^{m}\left|U_{i}\right| \leq m \frac{|V|}{|K|}
$$

that implies $m>|K|$.

Lemma 4. Let $U_{1}, \ldots, U_{r}, V_{1}, \ldots, V_{s}$ be different spaces over $K$. Assume that $a_{1}, \ldots, a_{r}, b_{1}, \ldots, b_{s}>0$ and

$$
\sum_{i=1}^{r} a_{i} \mathbb{1}_{U_{i}}=\sum_{i=1}^{s} b_{i} \mathbb{1}_{V_{i}}
$$

Then $\max \{r, s\}$ is greater than the cardinality of $K$.

Proof. Among the spaces $V_{1}, \ldots, V_{s}, U_{1}, \ldots, U_{r}$ choose one that is maximal under inclusion. It is either $V_{i}$ for some $i \in\{1, \ldots, s\}$, or $U_{j}$ for some $j \in\{1, \ldots, t\}$. In the first case $V_{i}=\bigcup_{j=1}^{r}\left(V_{i} \cap U_{j}\right)$, where for all $j \in\{1, \ldots, r\}, V_{i} \cap U_{j} \subset V_{i}$. From Lemma 3, $r>|K|$. Similarly, in the second case $s>|K|$.

Theorem 3. Let $L$ be a finite field and let $K$ be a proper subfield of $L$. Let $m \leq|K|$ and let $C$ be a $K$-linear code in $L^{m}$. Any $K$-linear code isometry is extendible. Moreover, for any $m>|K|$ there exists a code in $L^{m}$ that has an unextendible $K$-linear isometry.

Proof. Assume that there exist a $K$-linear code $C \subseteq L^{m}$ and an unextendible $K$ linear isometry $f: C \rightarrow L^{m}$. Let $(\mathcal{U}, \mathcal{V})$ be two tuples of spaces that correspond to some basis of $C$ and the map $f$. Since $f$ is an isometry, Proposition 5 implies that eq. (1) holds and hence $(\mathcal{U}, \mathcal{V})$ is a solution. By Proposition 2, the solution $(\mathcal{U}, \mathcal{V})$ is nontrivial. Grouping equal terms on each side of eq. (1) we get,

$$
\sum_{i=1}^{r} a_{i} \mathbb{1}_{V_{i}^{\prime}}=\sum_{i=1}^{s} b_{i} \mathbb{1}_{U_{i}^{\prime}},
$$


where $V_{i}^{\prime}, U_{j}^{\prime}$ are $K$-linear spaces, $a_{i}, b_{j}>0$, for $i \in\{1, \ldots, r\}, j \in\{1, \ldots, s\}$, the spaces $V_{i}^{\prime}$, for $i \in\{1, \ldots, r\}$, are all different and the spaces $U_{i}^{\prime}$, for $i \in\{1, \ldots, s\}$ are all different. Note that $r, s \leq m$. Eliminate equal terms from different sides and make a renumbering of the spaces on both sides of the equation. The resulting equation is the following,

$$
\sum_{i=1}^{r^{\prime}} a_{i}^{\prime} \mathbb{1}_{V_{i}^{\prime \prime}}=\sum_{i=1}^{s^{\prime}} b_{i}^{\prime} \mathbb{1}_{U_{i}^{\prime \prime}},
$$

where $V_{i}^{\prime \prime}, U_{j}^{\prime \prime}$ are $K$-linear spaces, $a_{i}^{\prime}, b_{j}^{\prime}>0$, for $i \in\left\{1, \ldots, r^{\prime}\right\}, j \in\left\{1, \ldots, s^{\prime}\right\}$, and the spaces $V_{i}^{\prime \prime}, U_{j}^{\prime \prime}$, for $i \in\left\{1, \ldots, r^{\prime}\right\}, i \in\left\{1, \ldots, s^{\prime}\right\}$ are all different. In the last equation, all the conditions of Lemma 4 are satisfied and therefore $\max \left\{r^{\prime}, s^{\prime}\right\}>|K|$. Note that $r^{\prime} \leq r \leq m$ and $s^{\prime} \leq s \leq m$. Therefore $m>|K|$.

For $m=|K|+1$ we have already introduced a $K$-linear code in $L^{m}$ with unextendible $K$-linear isometry in Example 2. Evidently, for $m>|K|+1$ such a pair of codes and an isometry is constructed by adding a set of arbitrary columns to the generator matrices of the two codes from Example 2.

Of course, the techniques developed in the paper can be used to prove the classical MacWilliams Extension Theorem for linear codes. For the case $K=L$ we can refine Theorem 3 .

Proof of the MacWilliams Extension Theorem. Due to Proposition 2 and Proposition 5, where the field $K$ is considered to be $L$, it is enough to show that all solutions $(\mathcal{U}, \mathcal{V})$ of eq. $(1)$ are trivial. By the definition of column space, for all $i \in\{1, \ldots, m\}, \operatorname{dim}_{K} V_{i} \leq n$ and $\operatorname{dim}_{K} U_{i} \leq n$, where $n=[L: K]=1$. Therefore the spaces in $\mathcal{U}$ and $\mathcal{V}$ are just one-dimensional or zero spaces and hence a solution of eq. (1) can be only trivial.

It is worth to note that, except the case $K=L$, in the paper we never used the fact that $L$ is a field. We only required $L$ to be a vector space over $K$. As we mentioned above, the character techniques allows the generalization of properties of codes over fields to the case of codes over rings and over modules. The generalization of Proposition 2 and Proposition 5 to the case of codes linear over modules is possible and will appear in our further works.

\section{Unextendible additive isometries}

In this section we give a description of one family of nontrivial solutions of eq. (1) in the case of $m=q+1$, where by $q$ we denoted the cardinality of the field $K$. As we mentioned above, nontrivial solutions of eq. (1) are in the correspondence with unextendible $K$-linear code isometries (see Proposition 2 and Proposition 5).

In Lemma 3 we proved that the covering of a space by proper subspaces is possible only if the number of subspaces is not less than $q+1$. The following lemma gives the description all such possible covering.

Lemma 5. Let $V$ be a vector space over $K$ of dimension $k \geq 2$. Let $U_{i}, i \in$ $\{1, \ldots, q+1\}$ be proper subspaces of $V$. If $V=\bigcup_{i=1}^{q+1} U_{i}$, then there exists a subspace $S \subset V$ of dimension $k-2$ such that $\left\{U_{1}, \ldots, U_{q+1}\right\}$ is the set of all subspaces of dimension $k-1$ that contain $S$. 
Proof. Assume that there are at least two spaces, let them be $U_{q}$ and $U_{q+1}$, with dimensions smaller than $k-1$. Then $q^{k}=|V|=\left|\bigcup_{i=1}^{q+1} U_{i}\right|<\sum_{i=1}^{q-1}\left|U_{i}\right|+$ $\left|U_{q}\right|+\left|U_{q+1}\right| \leq(q-1) q^{k-1}+2 q^{k-2}=q^{k}-q^{k-1}+2 q^{k-2}$, which is not true since $q \geq 2$. Therefore there exists at most one space $U_{i}$ with $\operatorname{dim}_{K} U_{i} \leq k-2$, $i \in\{1, \ldots, q+1\}$. Assume it exists and let it be $U_{q+1}$. For $i \in\{2, \ldots, q+$ $1\}$ define a set $\bar{U}_{i}=\bigcup_{j<i} U_{j}$ and notice that for $i \in\{2, \ldots, q\},\left|U_{i} \backslash \bar{U}_{i}\right| \leq$ $\left|U_{i}\right|-\left|U_{i} \cap U_{1}\right|=q^{k-1}-q^{k-2}$, because $\operatorname{dim}_{K} U_{1} \cap U_{i}=k-2$. The equality $\left|U_{i} \backslash \bar{U}_{i}\right|=q^{k-1}-q^{k-2}$ holds if and only if $U_{i} \cap \bar{U}_{i}=U_{i} \cap U_{1}$, where $i \in\{2, \ldots, q\}$. Obviously, $\left|U_{q+1} \backslash \bar{U}_{q+1}\right| \leq q^{k-2}-1$. In the equality $V=U_{1} \cup \bigcup_{i=2}^{q+1}\left(U_{i} \backslash \bar{U}_{i}\right)$ all sets in the union are disjoint. Thus

$$
q^{k}=|V|=\left|U_{1}\right|+\sum_{i=2}^{q+1}\left|U_{i} \backslash \bar{U}_{i}\right| \leq q^{k-1}+(q-1)\left(q^{k-1}-q^{k-2}\right)+q^{k-2}-1 .
$$

Regrouping the terms we get $2 q^{k-2} \geq q^{k-1}+1$, which gives a contradiction. Hence, $\operatorname{dim}_{K} U_{i}=k-1$ for all $i \in\{1, \ldots, q+1\}$ and we can refine the inequality,

$$
q^{k}=|V|=\left|U_{1}\right|+\sum_{i=2}^{q+1}\left|U_{i} \backslash \bar{U}_{i}\right| \leq q^{k-1}+q\left(q^{k-1}-q^{k-2}\right)=q^{k} .
$$

This implies that for all $i \in\{2, \ldots, q+1\},\left|U_{i} \backslash \bar{U}_{i}\right|=q^{k-1}-q^{k-2}$ and therefore $U_{i} \cap \bar{U}_{i}=U_{i} \cap U_{1}$. Define the space of dimension $k-2, S=U_{2} \cap U_{1}$. The following equalities hold, $U_{1} \cap U_{i}=U_{i} \cap \bar{U}_{i}=U_{2} \cap\left(U_{i} \cap \bar{U}_{i}\right)=U_{2} \cap\left(U_{i} \cap U_{1}\right)=U_{i} \cap S$, for all $i \in\{2, \ldots, q+1\}$. So $U_{i} \cap S$ has dimension $k-2$, which implies $S \subset U_{i}$, for all $i \in\{2, \ldots, q+1\}$. Evidently, the spaces $U_{i}$ for $i \in\{1, \ldots, q+1\}$ include all the spaces that are strictly between $S$ and $V$.

For a pair of spaces $S \subset V$ of dimensions $n-2$ and $n$ correspondingly define two tuples of spaces $\mathcal{U}^{A}=\left(U_{1}^{A}, \ldots, U_{q+1}^{A}\right)$ and $\mathcal{V}^{A}=\left(V_{1}^{A}, \ldots, V_{q+1}^{A}\right)$ in the following way. Let $V_{1}^{A}=\cdots=V_{q}^{A}=V, V_{q+1}^{A}=S$ and let $U_{1}^{A}, \ldots, U_{q+1}^{A}$ be all different hyperplanes in $V$ that contain $S$.

Proposition 6. Let $\mathcal{U}$ and $\mathcal{V}$ be two tuples of spaces such that

$$
\max _{i \in\{1, \ldots, q+1\}} \operatorname{dim}_{K} V_{i}>\max _{i \in\{1, \ldots, q+1\}} \operatorname{dim}_{K} U_{i} .
$$

The pair $(\mathcal{U}, \mathcal{V})$ is a nontrivial solution of eq. (1) if and only if there exist spaces $V$ and $S$ of dimension $k$ and $k-2$ correspondingly, such that $\mathcal{U} \sim \mathcal{U}^{A}$ and $\mathcal{V} \sim \mathcal{V}^{A}$

Proof. Prove the only if part. Without loss of generality, assume that $\operatorname{dim}_{K} V_{1}=$ $k=\max _{i \in\{1, \ldots, q+1\}} \operatorname{dim}_{K} V_{i}$. Obviously, $k \geq 2$ and from eq. (1), $V_{1}=\bigcup_{i=1}^{q+1}\left(U_{i} \cap\right.$ $V_{1}$ ), where $U_{i} \cap V_{1} \subset V_{1}$ for all $i \in\{1, \ldots, q+1\}$. From Lemma 5 there exists a subspace $S \subset V_{1}$ such that $\operatorname{dim}_{K} S=k-2$ and $S \subset U_{i} \cap V_{1} \subset V_{1}$, $\operatorname{dim}_{K} U_{i} \cap V_{1}=k-1$ and all the spaces $U_{i} \cap V_{1}$ are different for $i \in\{1, \ldots, q+1\}$. From the conditions $\operatorname{dim}_{K} U_{i} \cap V_{1}=k-1, \operatorname{dim}_{K} U_{i}<k$ and $U_{i} \cap V_{1} \subset V_{1}$ we deduce $U_{i}=U_{i} \cap V_{1} \subset V_{1}$, where $i \in\{1, \ldots, q+1\}$. Since $V_{1}=\bigcup_{i=1}^{q+1} U_{i}$ it is easy to see that $\mathbb{1}_{V_{1}}+q \mathbb{1}_{S}=\sum_{i=1}^{q+1} \mathbb{1}_{U_{i}}$. Equation (1) can be rewritten as

$$
\frac{1}{q^{k}} \mathbb{1}_{V_{1}}+\sum_{i=2}^{q+1} \frac{1}{\left|V_{i}\right|} \mathbb{1}_{V_{i}}=\frac{1}{q^{k-1}} \sum_{i=1}^{q+1} \mathbb{1}_{U_{i}}=\frac{1}{q^{k-1}} \mathbb{1}_{V_{1}}+\frac{1}{q^{k-2}} \mathbb{1}_{S}
$$


Subtracting $q^{-k} \mathbb{1}_{V_{1}}$ from both sides we get

$$
\sum_{i=2}^{q+1} \frac{1}{\left|V_{i}\right|} \mathbb{1}_{V_{i}}=\frac{q-1}{q^{k}} \mathbb{1}_{V_{1}}+\frac{1}{q^{k-2}} \mathbb{1}_{S} .
$$

Since $q>1, V_{1}=\bigcup_{i=2}^{q+1}\left(V_{i} \cap V_{1}\right)$. From Lemma 5, considering the fact that the number of terms from both sides is less than $q+1$, there exists $i \in\{2, \ldots, q+1\}$ such that $V_{i}=V_{1}$. Assume $V_{2}=V_{1}$ and reduce the equation,

$$
\sum_{i=3}^{q+1} \frac{1}{\left|V_{i}\right|} \mathbb{1}_{V_{i}}=\frac{q-2}{q^{k}} \mathbb{1}_{V_{1}}+\frac{1}{q^{k-2}} \mathbb{1}_{S} .
$$

Repeating the procedure $q-2$ more times we get that $V_{i}=V_{1}$ for every $i \in$ $\{1, \ldots, q\}$ and the reduced equation becomes $\frac{1}{\left|V_{q+1}\right|} \mathbb{1}_{V_{q+1}}=\frac{1}{q^{k-2}} \mathbb{1}_{S}$. Obviously, $V_{q+1}=S$. Defining $V=V_{1}$, we proved that $\mathcal{U} \sim \mathcal{U}^{A}$ and $\mathcal{V} \sim \mathcal{V}^{A}$.

In the other direction, easy to see that the pair $\left(\mathcal{U}^{A}, \mathcal{V}^{A}\right)$ is really a solution of eq. (1).

Having a family of nontrivial solutions for $m=q+1$ we can build a family of unextendible $K$-linear code isometries for codes of length $q+1$. The unextendible additive isometry presented in Example 3 is a particular case, which corresponds to the solution $\left(\mathcal{U}^{A}, \mathcal{V}^{A}\right)$ with $V=K^{2}$ and $S=\{0\}$.

The full description of nontrivial solutions of eq. (1) will appear in the further paper.

\section{References}

[1] I. Constantinescu and W. Heise, "On the concept of code-isomorphy," Journal of Geometry, vol. 57, no. 1-2, pp. 63-69, 1996.

[2] S. V. Avgustinovich and F. I. Solov'eva, "To the metrical rigidity of binary codes," Probl. Inf. Transm., vol. 39, pp. 178-183, Apr. 2003.

[3] F. Solov'eva, T. Honold, S. Avgustinovich, and W. Heise, "On the extendability of code isometries," Journal of Geometry, vol. 61, no. 1-2, pp. 2-16, 1998.

[4] D. I. Kovalevskaya, "On metric rigidity for some classes of codes," Probl. Inf. Transm., vol. 47, pp. 15-27, Mar. 2011.

[5] F. MacWilliams and N. Sloane, The Theory of Error-Correcting Codes: Vol.: 1. NorthHolland Mathematical Library, North-Holland Publishing Company, 1977.

[6] J. Gruska, Quantum Computing. McGraw-Hill Publishing Company, 1999.

[7] H. N. Ward and J. A. Wood, "Characters and the equivalence of codes," Journal of Combinatorial Theory, Series A, vol. 73, no. 2, pp. 348 - 352, 1996.

[8] M. Greferath, A. Nechaev, and R. Wisbauer, "Finite quasi-frobenius modules and linear codes," Journal of Algebra and Its Applications, vol. 03, no. 03, pp. 247-272, 2004.

[9] J. A. Wood, "Foundations of linear codes defined over finite modules: the extension theorem and the MacWilliams identities," in Codes over rings, vol. 6 of Ser. Coding Theory Cryptol., pp. 124-190, World Sci. Publ., Hackensack, NJ, 2009.

[10] J. Wood, "Extension theorems for linear codes over finite rings," in Applied Algebra, Algebraic Algorithms and Error-Correcting Codes (T. Mora and H. Mattson, eds.), vol. 1255 of Lecture Notes in Computer Science, pp. 329-340, Springer Berlin Heidelberg, 1997.

[11] S. Lang, Algebra. Addison-Wesley series in mathematics, Addison-Wesley Publishing Company, Advanced Book Program, 1984.

[12] J. A. Wood, "Exotic automorphisms of additive codes," AMS sectional meeting, Louisville, Kentucky, 2013.

[13] P. L. Clark, "Covering numbers in linear algebra.," The American Mathematical Monthly, vol. 119, no. 1, pp. 65-67, 2012. 